\title{
EFFECTS OF B CHROMOSOMES ON DEVELOPMENT IN GRASSHOPPER EMBRYOS
}

\author{
G. M. HEWITT and T. M. EAST \\ School of Biological Sciences, University of East Anglia, Norwich NR4 7TJ
}

Received 9.v.78

\begin{abstract}
SUMmary
Pre- and post-diapause embryonic development was described in Myrmeleotettix maculatus in 24 stages up to hatching. The rate of development and mitotic index of $0 \mathrm{~B}, 1 \mathrm{~B}$ and $2 \mathrm{~B}$ karyotypes in males and females were compared in the laboratory in material from two disjunct locations. The overall influence of one B chromosome on development does not appear to be major; however, higher numbers of Bs have a more noticeable effect. The B chromosome quite clearly slows down the early prediapause development of male embryos, and individuals with $2 \mathrm{~B}$ or $3 \mathrm{~B}$ chromosomes are on average retarded in the later stages of the post-diapause period prior to hatching. The presence of $\mathrm{B}$ chromosomes produces a variety of chromosomally mutant embryos, many of which are arrested prior to diapause and few develop to hatching. The results are discussed in relation to the maintenance of the $\mathrm{B}$ chromosome polymorphism in this insect.
\end{abstract}

\section{INTRODUGTION}

Populations of the heathland grasshopper Myrmeleotettix maculatus from most parts of southern Britain exhibit a stable polymorphism for a large supernumerary chromosome. This B chromosome has not been found in other parts of Britain nor in the adjacent continental countries of France and Belgium (Hewitt, 1973b).

In attempting to understand the maintenance of this polymorphism it has been demonstrated that: (1) a close correlation exists with microclimate across two steep clines in B frequency in Wales and East Anglia (Hewitt and Brown, 1970; Hewitt and Ruscoe, 1971; Robinson, 1973); (2) the frequency of the $\mathrm{B}$ chromosome undergoes regular changes during the embryonic and nymphal development in the three populations investigated (Robinson and Hewitt, 1976); (3) the B chromosome is inherited through the female with meiotic drive and through the male with " meiotic drag" (Hewitt, 1973a, 1976); (4) its presence in an individual increases chiasma frequency and variance (John and Hewitt, 1965; Barker, 1966; Hewitt and John, 1967, 1970).

Several hypotheses could explain these data, but the stability of the population frequencies from year to year and the regular fluctuation in frequency during each life cycle clearly suggest that selective forces are acting between the grasshoppers with different karyotypes. However, previous work with B chromosomes in other animal and plant species has shown their phenotypic effects, if any, to be slight and of a quantitative nature (see Jones, 1975, for references). Despite considerable effort, as yet no morphological effect of the $\mathrm{B}$ chromosome has been discovered in $M$. maculatus (Hewitt and John, 1970; Harvey, 1977). The changes in B 
frequency in natural populations during the egg stage mentioned above are quite marked and it is therefore an obvious and relevant question to ask if there is any detectable effect of the B chromosome on embryo development.

\section{Materials AND Methods}

A pilot run was undertaken using grasshoppers from East Anglia. The results of this were encouraging and led to the experiments reported here. For these main experiments young adult grasshoppers were collected from two stable, abundant and well-known populations at Foxhole (East Anglia) and Talybont (West Wales). These were kept in cages in the insectary and the sand in the oviposition trays sieved each day for egg pods. About half of the pods were opened, the eggs plated on to moist filter paper (Stebbings and Hewitt, 1972, for technique) and used for pre-diapause observations. The remainder of the pods were placed whole in moist sand and allowed to complete their pre-diapause development in an incubator at $26^{\circ} \mathrm{C}$.

The eggs plated for pre-diapause work were sampled over 26 days after laying, care being taken to sample eggs from the same pod on different days through the pre-diapause period. The eggs sampled were dissected and the embryos cultured in hypotonic saline with 0.05 per cent colchicine for $30 \mathrm{~min}$. prior to fixation in $3: 1$ alcohol : acetic. A record was kept of the size and form of each embryo by which they were allocated to a developmental stage (fig. 1). The karyotype of each embryo was determined from orcein squash preparations along with the mitotic index, i.e. per cent cells in mitosis. This latter measure was obtained from 1000 cells with no more than 100 cells from any one field of view in order to adequately sample all the embryonic tissues.
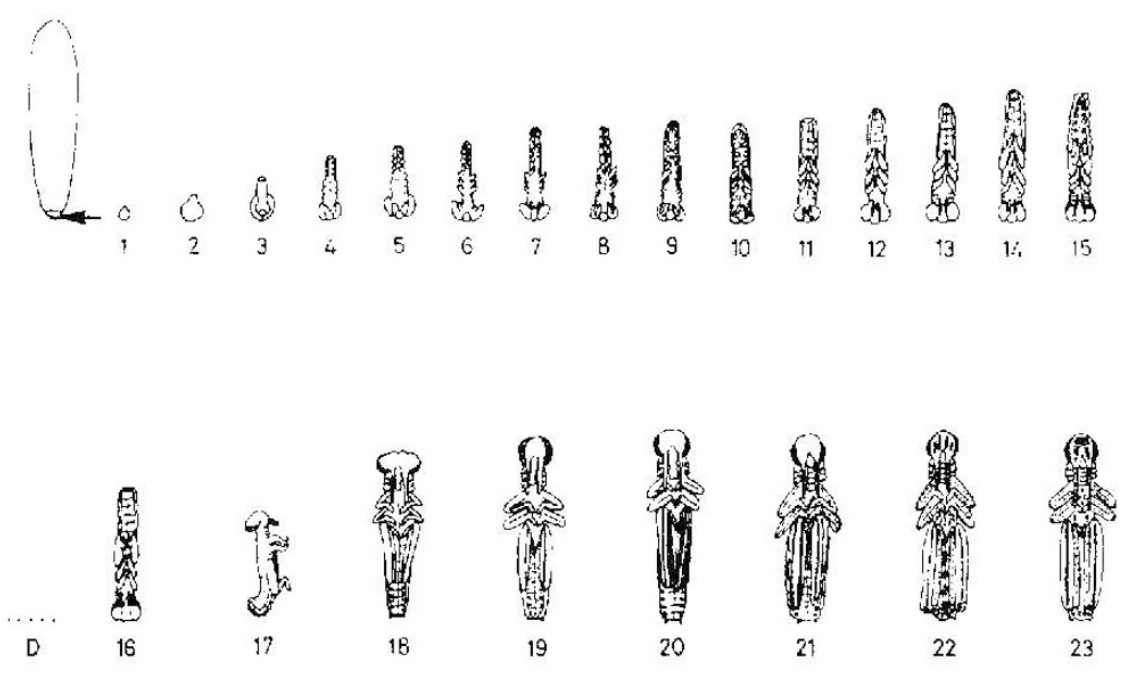

Fig. 1.-Drawings of the stages in embryonic development of Myrmeleotettix maculatus recognised from this study. The micropylar end of the egg points down with an arrow indicating the location of the oocyte nucleus and zygote. We define 15 stages up to diapause (D) and eight post-diapause; blastokinesis occurs at stage 17 and hatching occurs at stage 24 . 
The pods allocated for post-diapause observations were allowed 4 weeks to complete their pre-diapause development and the trays of moist sand containing them were then placed at $4^{\circ} \mathrm{G}$ for 8 weeks to break the diapause. The pods were then opened and the eggs plated on moist filter paper, as for the pre-diapause eggs, and kept at $26^{\circ} \mathrm{C}$. Eggs were sampled each day for 14 days, the embryos were treated with colchicine, fixed, karyotyped and recorded as for the pre-diapause eggs.

\section{Results}

It is possible to describe the pre-diapause development of $M$. maculatus embryos in 14 or 15 stages (fig. 1). The developing embryo is usually too small to handle under 5 days of age when stage 1 is reached and this is a small mass of some 1000 cells. The mitotic index per individual in this early stage varies between 1 per cent and 6 per cent averaging 3 per cent; by stage 12 this average has dropped to 2 per cent and just prior to diapause it is 1.5 per cent. Embryos in diapause show no mitotic divisions; healthy diapause embryos have nuclei with deep staining, somewhat granular chromatin. Some embryos at stage 14 were in diapause, others reached stage 15 before ceasing cell division. These two stages are differentiated by the development of obvious eye pigmentation. The Foxhole embryos reached diapause about 3 days earlier on average than those from Talybont, i.e. 20 days and 23 days respectively.

Under our conditions, no obvious developmental changes occur in $M$. maculatus embryos during diapause; then, when the eggs are placed at $26^{\circ} \mathrm{C}$, hatching occurs after 12-13 days when the embryos are at stage 24 . The embryo undergoes blastokinesis, turning to face upwards in the egg, at stage 17 which is 2-3 days after removal from the cold. The Talybont embryos were on average further advanced than the Foxhole ones in these initial few days of post-diapause, and they hatched a day earlier. The average mitotic index was 0.5 per cent 1 day post-diapause, rose to 1.0 per cent at stage 17 , dropped to 0.2 per cent by stage 20 and stayed at or below $0 \cdot 1$ per cent from stage 21 to hatching.

Several grossly malformed embryos were found in the pre-diapause material, becoming particularly obvious between days 11 and 16 . In the Foxhole material 5 out of 208 embryos karyotyped were grossly malformed and all were numerical chromosome mutants. A further 11 embryos had numerically mutant karyotypes and although all were somewhat retarded in development they were of normal shape. Of the 306 Talybont prediapause embryos 16 were numerical chromosome mutants and all were retarded to a greater or lesser extent. These mutants were not included in the analyses.

Likewise a number of malformed or retarded embryos were found in the post-diapause material. All except one of the malformed embryos that could be karyotyped were numerical chromosome mutants, i.e. in Foxhole $3 / 230$, in Talybont 10/405. A further 12 retarded embryos from Foxhole were numerical chromosome mutants, and there were 19 embryos in this category from Talybont. No chromosomal mutants were included in the analyses since they are clearly associated with developmental malformations and retardation. It should be pointed out that of these numerical chromosome mutants some were with B chromosomes and some without, although 
the larger proportion had B chromosomes, viz. Foxhole 9 OB : $22+\mathrm{B}$, Talybont 15 0B : $20+B$.

A further category of embryos found in the post-diapause samples was also excluded from the analysis. These were apparently chromosomally normal but were still at pre-diapause stages (i.e. prior to stage 14) and had seemingly been arrested in development. At Foxhole there were $340 \mathrm{~B}: 13$ $+\mathrm{B}$ and at Talybont $60 \mathrm{~B}: \mathrm{l} 6+\mathrm{B}$.

The data on the stage, age, karyotype and mitotic index of pre- and post-diapause embryos from the Foxhole and Talybont populations is extensive involving some 1052 completely analysed embryos. For brevity, therefore, only the Foxhole data are presented graphically for stage/age, for sex and for B karyotype (figs. 2 and 3), and these may serve as an illustration.

The data were first investigated using analysis of covariance (Snedecor, 1956, p. 394). For each population they were subdivided into male and female, pre- and post-diapause, and the covariance of stage with age and mitotic index with age determined for different B karyotypes. One may then determine whether the linear regressions of embryo development are the same for different karyotypes, in particular if the slopes and the elevations (means) are significantly different for $\mathrm{OB} v .1 \mathrm{~B}$ and $\mathrm{OB} v .2 \mathrm{~B}$ embryos.

The results of these computations for the developmental stage reached at each age are presented in table 1 . The majority of these comparisons between $\mathrm{OB}$ and $\mathrm{B}$-containing embryos show no significant difference in the slope or elevation of the regressions for developmental rate. In general the embryonic development is little affected by the presence of one or two B chromosomes. However, the pre-diapause development of males is modified

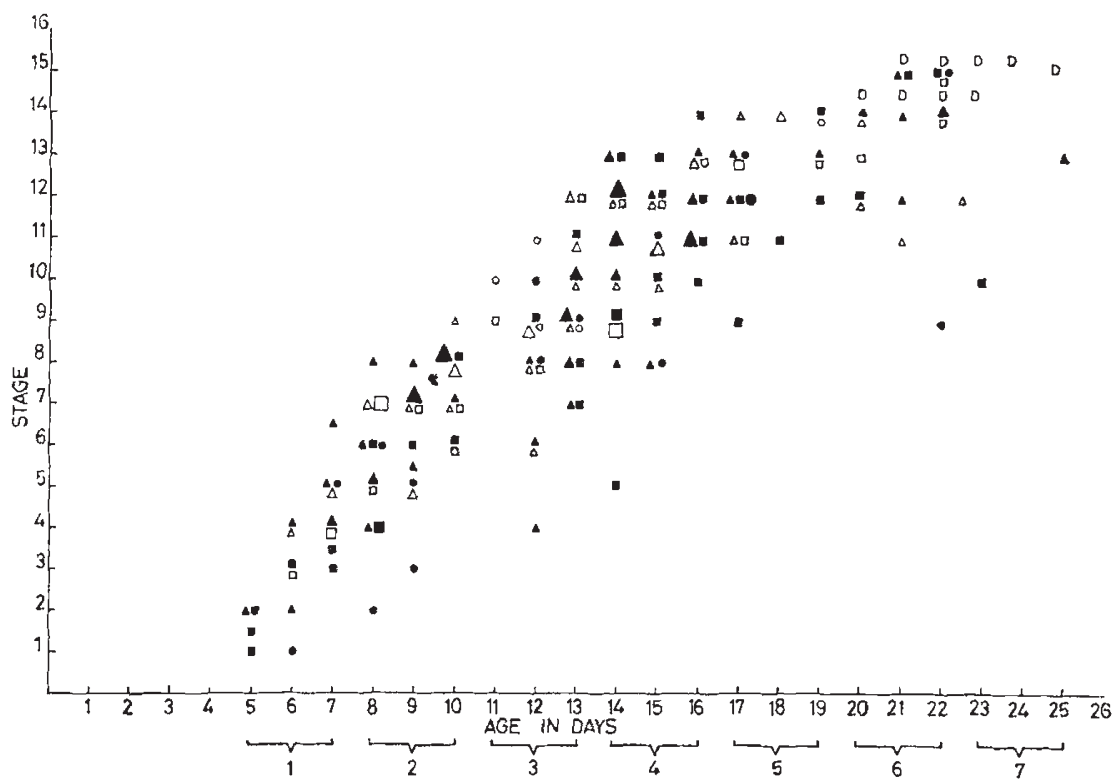

FIG. 2.-A graph of embryo stage against age in the pre-diapause period from the Foxhole population. The solid symbols are males, the open symbols are females. Triangles are $0 \mathrm{Bs}$, squares are $1 \mathrm{Bs}$ and circles are $2 \mathrm{Bs}$ or more. The smallest-size symbol equals one individual and progressively larger ones equal two, three, four or five individuals. Age classes are shown at the bottom, each of 3 days. 


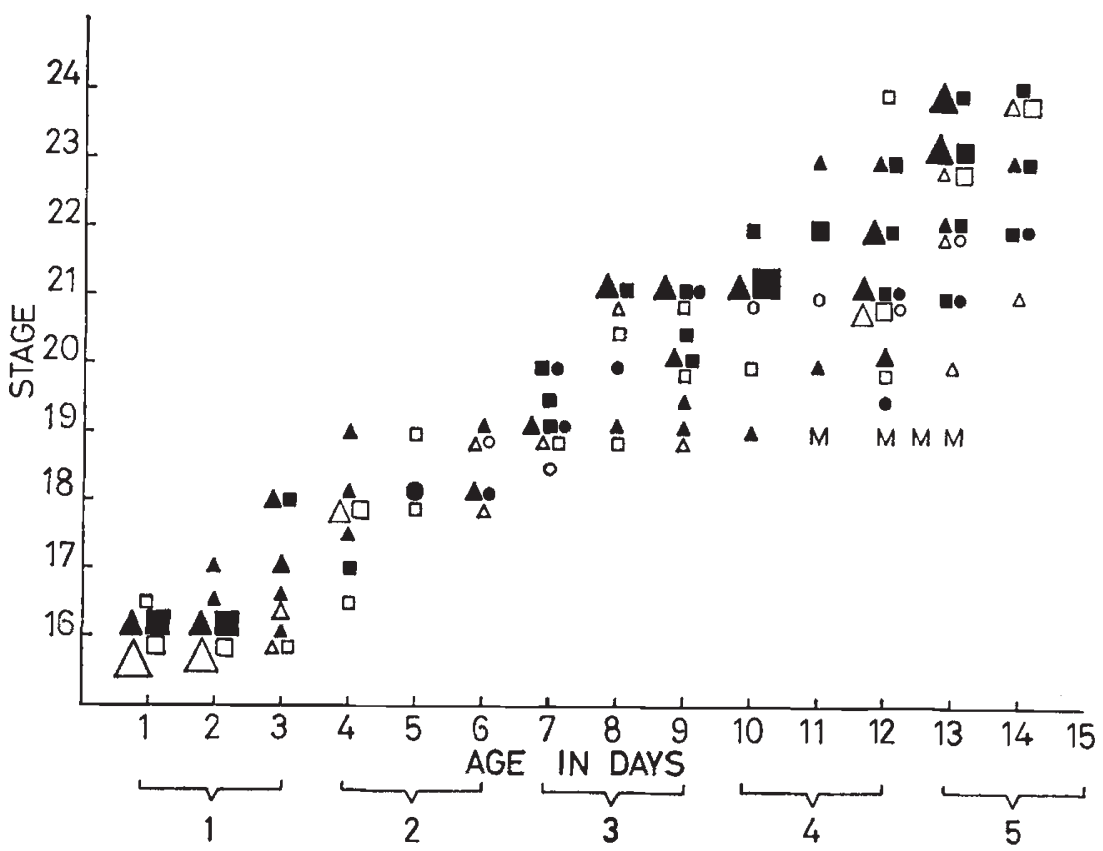

FIG. 3.-A graph of embryo stage against age in the post-diapause period for the Foxhole population. Symbols as in fig. 2. $M=$ chromosomally mutant embryos.

\section{TABLE 1}

Summary of the results of covariance analysis. (a) Figures in the table are significance levels and probability values from variance ratios to test if the slopes or elevations of the regressions of stage/age for $O B, 1 B$ and $2 B$ embryos are different (see Snedecor, 1956, p. 394). The occasional $3 B$ individual was included with the $2 B$ class. (b) The same analysis for mitotic index/age; only the relevant parts of the results are shown, the remaining comparisons being not formally significant.

(a) Stage/age

\begin{tabular}{|c|c|c|c|c|c|}
\hline & \multicolumn{2}{|c|}{ Foxhole } & \multicolumn{2}{|c|}{ Talybont } \\
\hline & & Slope & Elevation & Slope & Elevation \\
\hline \multicolumn{6}{|l|}{ Males } \\
\hline Pre-diapause, & $\begin{array}{l}\mathrm{OB} v .1 \mathrm{~B} \\
\mathrm{OB} v .2 \mathrm{~B}\end{array}$ & $\begin{array}{l}0.01-0.001 \\
0.02-0.01\end{array}$ & $\begin{array}{l}0 \cdot 1-0 \cdot 05 \\
\text { Not sig. }\end{array}$ & $\begin{array}{l}0 \cdot 05-0.025 \\
\text { Not sig. }\end{array}$ & $\begin{array}{l}0.05-0.025 \\
0 \cdot 1-0.05\end{array}$ \\
\hline Post-diapause, & $\begin{array}{l}\mathrm{OB} v .1 \mathrm{~B} \\
\mathrm{OB} v .2 \mathrm{~B}\end{array}$ & $\begin{array}{l}\text { Not sig. } \\
0.05-0 \cdot 025\end{array}$ & $\begin{array}{l}\text { Not sig. } \\
0 \cdot 2-0 \cdot 1\end{array}$ & $\begin{array}{l}\text { Not sig. } \\
\text { Not sig. }\end{array}$ & $\begin{array}{l}\text { Not sig. } \\
0 \cdot 2-0 \cdot 1\end{array}$ \\
\hline
\end{tabular}

Females

$\begin{array}{llllll}\text { Pre-diapause, } & \text { OB } v .1 \mathrm{~B} & \text { Not sig. } & \text { Not sig. } & \text { Not sig. } & \text { Not sig. } \\ & \text { OB } v .2 \mathrm{~B} & \text { Not sig. } & \text { Not sig. } & \text { Not sig. } & 0 \cdot 2-0 \cdot 1 \\ \text { Post-diapause, } & \text { OB } v .1 \mathrm{~B} & 0 \cdot 1-0 \cdot 05 & \text { Not sig. } & 0 \cdot 2-0 \cdot 1 & \text { Not sig. } \\ & \text { OB } v .2 \mathrm{~B} & \text { Not sig. } & \text { Not sig. } & \text { Not sig. } & \text { Not sig. }\end{array}$

Males

(b) Mitotic index/age

$\begin{array}{llllll}\text { Pre-diapause } & \text { OB } v .1 \mathrm{~B} & \text { Not sig. } & 0 \cdot 1 & \text { Not sig. } & 0 \cdot 025-0.01 \\ & \text { OB } v .2 \mathrm{~B} & \text { Not sig. } & \text { Not sig. } & \text { Not sig. } & 0 \cdot 05-0.025\end{array}$


significantly by the presence of $\mathrm{B}$ chromosomes. It is evident from the data that the $\mathrm{OB}$ individuals are developing faster than the $\mathrm{B}$ individuals in the early stages. One means of achieving this advance could be through a higher mitotic rate; the covariance analysis of mitotic index with age showed only the Talybont data as significant, with the $0 \mathrm{Bs}$ having a higher mitotic rate than the Bs. The same trend was not significant for Foxhole embryos (table 1). As was indicated previously there is considerable variation in mitotic index between embryos at the same stage and this will tend to obscure any differences. No other covariance analysis of mitotic index with age showed a significant difference between $\mathrm{OB}$ and $\mathrm{B}$ embryos.

The covariance analyses of stage with age also indicate a difference in the post-diapause period between $\mathrm{OB}$ and $2 \mathrm{~B}$ male embryos; this reaches formal significance in the Foxhole data. It would seem that the development of $2 \mathrm{~B}$ and $3 \mathrm{~B}$ males slows down towards the end of the post-diapause period.

In order to locate more precisely the developmental stages at which these differences between $\mathrm{OB}$ and $\mathrm{B}$ embryos occur and to test their statistical significance a series of analyses of variance were performed. For this the data were subdivided into 3 -day blocks beginning at day 5 (i.e. age class $1=$ days 5,6 and 7 ; class $2=8,9$ and 10 ; etc.). This gives seven age classes for pre-diapause and five for post-diapause development (see figs. 2 and 3). Three types of one-way analysis were used, viz. OB $v .1 \mathrm{~B}, 0 \mathrm{~B} v .1 \mathrm{~B} v$. $2 \mathrm{~B}$ and $\mathrm{OB}+1 \mathrm{~B} v .2 \mathrm{~B}$; in pre-diapause age class 1 there were not really enough embryos to test effectively for the two sexes separately. The results are summarised in table 2 .

The difference in developmental rate noticed for males early in prediapause is confirmed by this analysis and is quite marked in the Foxhole material. At the end of pre-diapause the Talybont male embryos with Bs are behind their $0 \mathrm{~B}$ fellows. Likewise the tardiness of the $2 \mathrm{~B}$ embryos is also evident towards the end of the post-diapause period as hatching is approached in both the Foxhole and Talybont material.

The mitotic indices of Talybont pre-diapause $0 \mathrm{~B}, 1 \mathrm{~B}$ and $2 \mathrm{~B}$ embryos were different in two phases of development, the $0 \mathrm{Bs}$ having the higher indices. This also confirms the results of the covariance analysis.

\section{Disaussion}

These data and analyses clearly demonstrate that B chromosomes modify the development of male embryos in the pre-diapause period. The major effect of this is seen in the early stages when B embryos are less well developed than $0 B$ ones. However, this does not lead to any detectable difference between the karyotypes in the speed with which diapause is reached, although the considerable variation between individuals would tend to mask any small distinctions. Egg samples taken from the field show that in the autumn when the embryos are just into diapause there is an increase in the B frequency over the parental generation; but the frequency of B chromosomes drops during the winter while the embroys are in diapause (Robinson and Hewitt, 1976). This autumnal pre-diapause increase could be due to meiotic drive in the female (Hewitt, 1976) and also selective differences between the karyotypes. However, this current work gives no indication of any major detriment caused by the B chromosome during the pre-diapause period. It is of course possible that retardation early in development may 


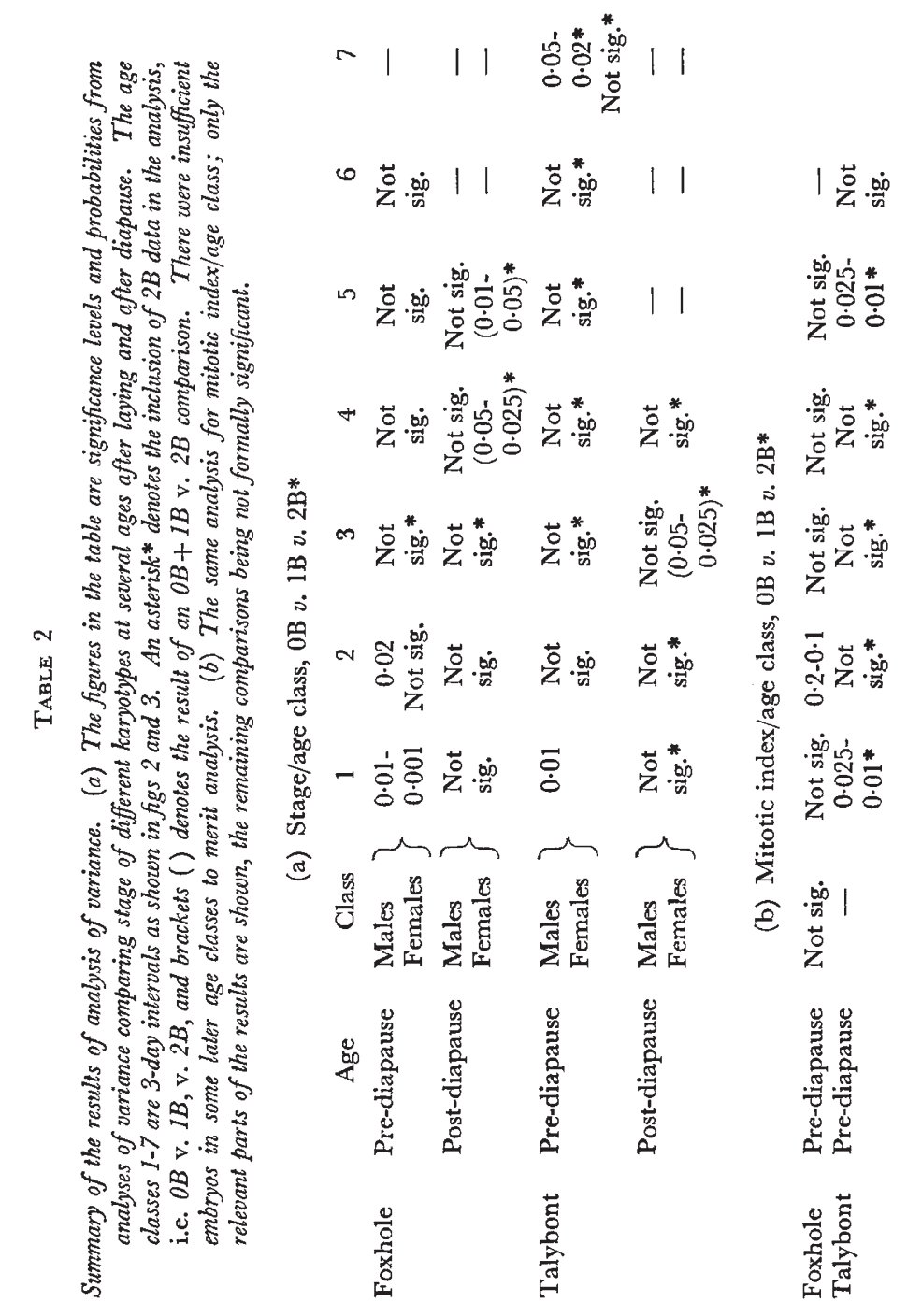


affect the physiological state in which an embryo enters diapause and hence its ability to survive through the winter. It is peculiar that the male embryos are affected while the females are apparently not. There are also indications of such a sexual difference in the effect of B chromosomes in the nymphal development of this species (Harvey, 1977). Conversely Procunier (1975) showed that B chromosomes are correlated with slow development in females of the Blackfly Cnephia dacotensis, while in males the effect is not obvious.

Just why the development of one sex should be affected by the B chromosome is unclear. However, the following three points are possibly relevant. One may readily observe in the field that $M$. maculatus males develop to fledging several days faster than females and this has been demonstrated in the laboratory under controlled conditions (Harvey, 1977). The male is the heterogametic sex-the sex chromosome system being $\mathrm{XO}$ o : $\mathrm{XX}$ 우. It is possible that the $\mathrm{B}$ chromosome is derived from the $\mathrm{X}$ chromosome (Hewitt, 1973b).

The second result to emerge is that towards the end of the post-diapause period, embryos with 2 or 3Bs are on average retarded in their development. In his studies of nymphal development Harvey (1977) also demonstrated that $2 \mathrm{~B}$ and $3 \mathrm{~B}$ individuals were slower to reach fledging than $\mathrm{OB}$ and $\mathrm{BB}$ individuals. One would expect that under most circumstances this would reduce their contribution to the next generation. The fitness of these karyotypes is also reduced by the effects of two (or more) B chromosomes on female and male meiosis so that aneuploid embryos and abnormal sperm are produced (Hewitt, 1973a and $b$ ). This result is in keeping with the general finding from $\mathrm{B}$ chromosome systems, that larger numbers of Bs reduce the fitness of those individuals carrying them (see Jones, 1975, for review).

The $\mathrm{B}$ chromosome in $M$. maculatus does not have any clear effect on the rate of cell division as measured by mitotic index. There are significantly lower mitotic indices for B embryos at some stages in the Talybont prediapause data, but not in the Foxhole data. However, there is a higher frequency of $\mathrm{B}$ chromosome in the Talybont population and the range of mitotic indices at most stages in both sets of data is considerable; these two factors may explain the apparent difference in the results from the two populations. One might expect that a reduced rate of development could be attained through a lower rate of cell division. Thus the mitotic cycle time is increased by B chromosomes in the plants Secale cereale (Ayonoadu and Rees, 1968) and Puschkinia libanotica (Barlow, 1973). Further work is required on this aspect in $M$. maculatus.

As mentioned above B chromosomes increase the production of chromosomally mutant embryos (Hewitt, 1973), and both Foxhole and Talybont embryos contained a variety of chromosomal mutants. Polyploids, multiple aneuploids, large chromosome trisomics and particularly monosomics were malformed and somewhat retarded in pre-diapause. The majority of postdiapause chromosomal mutants were severely retarded and most seemed to be arrested at pre-diapause stages $8-14$, but some did reach the later stages approaching hatching (i.e. stage 19). More of the chromosome mutants possessed $\mathrm{B}$ chromosomes than not, as would be expected if a $\mathrm{B}$ chromosome in the parental genotype were increasing chromosomal variation in the gametes and progeny. Numerical chromosomal mutants are rarely found in nymphs and adults (Hewitt, 1973; Robinson, 1973; and unpublished). 
This means that the chromosomal mutants produced largely because of the B chromosome in these populations of $M$. maculatus generate a marked reduction in fecundity.

It is interesting to note that the Foxhole embryos reached diapause about three days earlier than those from Talybont; while in the post-diapause period those from Talybont were more advanced and reached hatching about 1 day before the Foxhole ones. The environmental conditions under which these two populations live are rather different; Talybont is in lowland West Wales with an earlier spring and Foxhole is in central East Anglia with a more continental climate. No detailed parallel observations have been made on the population dynamics in these two disjunct populations but the Talybont population usually appears further advanced when collections of young adults have been made at the end of June. One would expect the genotypes of the two populations to be adapted to suit different local environments.

Thus the overall effect of a B chromosome on embryo development in $M$. maculatus does not appear to be a drastic one, and this is in keeping with the general results from other systems (see Jones, 1975, for review). Indeed, despite considerable efforts involving multivariate analyses of many morphological measurements, no distinction could be made between $\mathrm{OB}$ and $\mathrm{BB}$ individuals (Hewitt and John, 1970; Harvey, 1977). However, in the grasshopper Camnula pellucida the B chromosome does shorten the femur and reduce the number of testicular follicles (Nur, 1969), and in the mealy bug Pseudococcus obscurus the tibia length was increased by increasing numbers of B chromosomes (Nur, 1962). Higher numbers of B chromosomes have more noticeable effects on development in $M$. maculatus than one supernumerary. These current results are of course from controlled conditions in the laboratory optimal for development; field conditions may exert different pressures and stresses on the developing embryo and this aspect deserves investigation.

Acknowledgements. - This work was financed by a grant from the Science Research Council to whom we are most grateful. Dr Eddie Arthur kindly advised us on statistical matters.

\section{REFERENCES}

AYONOADU, U. W., AND REES, H. 1968. The regulation of mitosis by B-chromosomes in rye. Exp. Cell Res., 52, 284-290.

BARKER, J. F. 1966. Climatological distribution of a grasshopper supernumerary chromosome. Evolution, 20, 665-667.

BARLow, P. w. 1973. In The Cell Cycle in Development and Differentiation, ed. M. Balls and F. S. Billet, pp. 133-165. Cambridge University Press, London.

HARVEY, A. w. 1977. The expression of supernumerary chromosomes in Myrmeleotettix maculatus (Thunberg) (Acrididae). Ph.D. thesis, University of East Anglia.

HEWITT, G. M. 1973a. Variable transmission rates of a B-chromosome in Myrmeloetettix maculatus (Thunb.) (Acrididae : Orthoptera). Chromosoma, 40, 83-106.

HEWITT, G. M. 1973b. The integration of supernumerary chromosomes into the Orthopteran genome. Cold Spring Harbor Symp. Quant. Biol., 38, 183-194.

hewIT, G. м. 1976. Meiotic drive for B-chromosomes in the primary oocytes of Myrmeleotettix maculatus (Orthoptera : Acrididae). Chromosoma, 56, 381-391.

HEWITT, G. M., AND BRoWN, F. M. 1970. The B-chromosome system of Myrmeleotettix maculatus. V. A steep cline in East Anglia. Heredity, 25, 363-371.

HEWITT, G. M., AND JOHN, B. 1967. The B-chromosome system of Myrmeleotettix maculatus (Thunb.) III. The statistics. Chromosoma, 21, 140-162. 
HewrTt, G. M., AND JoHn. B. 1970. The B-chromosome system of Myrmeleotettix maculatus (Thunb.). IV. The dynamics. Evolution, 24, 169-180.

HEWITT, G. M., AND RUSCOE, C. N. E. 1971. Changes in microclimate correlated with a cline for B-chromosomes in the grasshopper Myrmeleotettix maculatus. 7. Anim. Ecol., 40, 753-765.

John, B., AND HEWITT, G. M. 1965. The B-chromosome system of Myrmeleotettix maculatus (Thunb.). I. The mechanics. Chromosoma, 16, 548-578.

JONES, R. N. 1975. B-chromosome systems in flowering plants and animal species. Int. Rev. Cytol., 40, 1-100.

NUR, U. 1962. Population studies of supernumerary chromosomes in a mealy bug. Genetics, $47,1679-1690$.

NUR, U. 1969. Mitotic instability leading to an accumulation of B-chromosomes in grasshoppers. Chromosoma, 27, 1-19.

PROCUNIER, w. s. 1975. B-chromosomes of Cnephia dacotensis and C. ornithophilia (Diptera : Simuliidae). Can. F. Zool., 53, 1638-1647.

ROBINson, Р. м. 1973. The ecological genetics of the polymorphism for B-chromosomes in the grasshopper Myrmeleotettix maculatus (Thunb.) (Acrididae : Orthoptera). Ph.D. thesis, University of East Anglia.

ROBINSON, P. M., AND HEWTTT, G. M. 1976. Annual cycles in the incidence of B-chromosomes in the grasshopper Myrmeleotettix maculatus (Acrididae:Orthoptera). Heredity, 36, 399-412.

SNEDeCOR, G. W. 1956. Statistical Methods, 5th edition. Iowa State University Press.

STEBBings, A. F. K., AND HeWITT, G. M. 1972. The laboratory breeding of British Gomphocerine grasshoppers (Acrididae : Orthoptera). Acrida, 1, 233-245. 